Research Article

\title{
Graphic Representation of a Dimensional Expansion of Triangular Fuzzy Number
}

\author{
Yong Sik Yun \\ Department of Mathematics, Jeju National University, Jeju 63243, Republic of Korea \\ Correspondence should be addressed to Yong Sik Yun; yunys@jejunu.ac.kr
}

Received 24 June 2021; Accepted 15 July 2021; Published 26 July 2021

Academic Editor: Qingkai Zhao

Copyright (@ 2021 Yong Sik Yun. This is an open access article distributed under the Creative Commons Attribution License, which permits unrestricted use, distribution, and reproduction in any medium, provided the original work is properly cited.

We calculate Zadeh's max-min composition operators for two 3-dimensional triangular fuzzy numbers. We prove that if the 3dimensional result is limited to 2 dimensions, it is the same as the 2-dimensional result, which is shown as a graph. Since a 3-dimensional graph cannot be drawn, the value of the membership function is expressed with color density. We cut a 3dimensional triangular fuzzy number by a perpendicular plane passing a vertex, and consider the cut plane as a domain. The value of the membership function for each point on the cut plane is also expressed with color density. The graph expressing the value of the membership function, defined in the plane as a 3-dimensional graph using the $z$-axis value instead of expressing with color density, is consistent with the results in the 2-dimensional case.

\section{Introduction}

Many results exist for Zadeh's max-min composition operators. The results for triangular fuzzy numbers are well known [1-5]. We have generalized 1-dimensional triangular fuzzy number to 2-dimensional triangular fuzzy number and calculated Zadeh's max-min composition operators for 2dimensional fuzzy numbers [6]. In the 1-dimensional case, Zadeh's max-min composition operator can be calculated using $\alpha$-cuts. By defining parametric operations between two region valued $\alpha$-cuts, we obtained parametric operations for two triangular fuzzy numbers defined on $\mathbb{R}^{2}$ [7]. In the case of 3-dimensional fuzzy numbers, the $\alpha$-cuts are subsets of $\mathbb{R}^{3}$. By defining parametric operations between two ellipsoids including interior valued $\alpha$-cuts, we calculated Zadeh's max-min composition operators for two 3-dimensional fuzzy numbers [8]. This will help facilitate further study of triangular fuzzy matrices $[1,3,9]$.

In this paper, we prove that Zadeh's max-min composition operators for two 3-dimensional triangular fuzzy numbers on $\mathbb{R}^{3}$ constitutes the generalization of Zadeh's max-min compositions for two 2-dimensional triangular fuzzy numbers on $\mathbb{R}^{2}$. In addition, by limiting the graph of the 3-dimensional result to the 2-dimensional case, we prove that the result expressed as a graph is consistent with the graph of the 2-dimensional result.

\section{Preliminaries}

We define $\alpha$-cut and $\alpha$-set of the fuzzy set $A$ on $\mathbb{R}$ with the membership function $\mu_{A}(x)$.

Definition 1 (see [10]). An $\alpha$-cut of the fuzzy number $A$ is defined by $A_{\alpha}=\left\{x \in \mathbb{R} \mid \mu_{A}(x) \geq \alpha\right\} \quad$ if $\alpha \in(0,1]$ and $A_{0}=c l\left\{x \in \mathbb{R} \mid \mu_{A}(x)>\alpha\right\}$. For $\alpha \in(0,1)$, the set $A^{\alpha}=\left\{x \in X \mid \mu_{A}(x)=\alpha\right\}$ is said to be the $\alpha$-set of the fuzzy set $A, A^{0}$ is the boundary of $\left\{x \in \mathbb{R} \mid \mu_{A}(x)=\alpha\right\}$, and $A^{1}=A_{1}$.

Following Zadeh, Dubois, and Prade, the extension principle is defined as follows.

Definition 2 (see [11-13]). The extended addition $A(+) B$, extended subtraction $A(-) B$, extended multiplication $A(\cdot) B$, and extended division $A(/) B$ are fuzzy sets with membership functions as follows. For all $x \in A$ and $y \in B$, 


$$
\mu_{A(*) B}(z)=\sup _{z=x * y} \min \left\{\mu_{A}(x), \mu_{B}(y)\right\}, \quad *=+,-,,, / .
$$

Definition 3 (see [7]). A fuzzy set $A$ with a membership function:

$$
\mu_{A}(x, y)= \begin{cases}1-\sqrt{\frac{\left(x-x_{1}\right)^{2}}{a^{2}}+\frac{\left(y-y_{1}\right)^{2}}{b^{2}},} & b^{2}\left(x-x_{1}\right)^{2}+a^{2}\left(y-y_{1}\right)^{2} \leq a^{2} b^{2} \\ 0, & \text { otherwise, }\end{cases}
$$

where $a, b>0$ is called the 2-dimensional triangular fuzzy number and denoted by $\left(a, x_{1}, b, y_{1}\right)^{2}$.

Let $A=\left(a, x_{1}, b, y_{1}\right)^{2}$. Then, the $\alpha$-cut $A_{\alpha}$ of a 2 -dimensional triangular fuzzy number $A$ is an interior of an ellipse in an $x y$-plane, including the boundary

$$
A_{\alpha}=\left\{(x, y) \in \mathbb{R}^{2} \mid\left(\frac{x-x_{1}}{a(1-\alpha)}\right)^{2}+\left(\frac{y-y_{1}}{b(1-\alpha)}\right)^{2} \leq 1\right\} .
$$

Theorem 1 (see [7]). Let $A$ be a continuous convex fuzzy number defined on $\mathbb{R}^{2}$, and $A^{\alpha}=\left\{(x, y) \in \mathbb{R}^{2} \mid \mu_{A}(x, y)=\alpha\right\}$ be the $\alpha$-set of $A$. Then, for all $\alpha \in(0,1)$, there exist continuous functions $f_{1}^{\alpha}(t)$ and $f_{2}^{\alpha}(t)$ defined on $[0,2 \pi]$ such that

$$
A^{\alpha}=\left\{\left(f_{1}^{\alpha}(t), f_{2}^{\alpha}(t)\right) \in \mathbb{R}^{2} \mid 0 \leq t \leq 2 \pi\right\} .
$$

If $A$ is a continuous convex fuzzy number defined on $\mathbb{R}^{2}$, then the $\alpha$-cut $A_{\alpha}$ is a closed convex subset in $\mathbb{R}^{2}$.

Definition 4 (see [7]). Let $A$ and $B$ be convex fuzzy numbers defined on $\mathbb{R}^{2}$ and

$$
\begin{aligned}
& A^{\alpha}=\left\{\left(f_{1}^{\alpha}(t), f_{2}^{\alpha}(t)\right) \in \mathbb{R}^{2} \mid 0 \leq t \leq 2 \pi\right\}, \\
& B^{\alpha}=\left\{\left(g_{1}^{\alpha}(t), g_{2}^{\alpha}(t)\right) \in \mathbb{R}^{2} \mid 0 \leq t \leq 2 \pi\right\},
\end{aligned}
$$

be the $\alpha$-sets of $A$ and $B$, respectively. For $\alpha \in(0,1)$, we define that the parametric addition $A(+)_{p} B$, parametric subtraction $A(-)_{p} B$, parametric multiplication $A(\cdot)_{p} B$, and parametric division $A(/)_{p} B$ of two fuzzy numbers $A$ and $B$ are fuzzy numbers that have their $\alpha$-sets as follows:

(1) $A(+)_{p} B:\left(A(+)_{p} B\right)^{\alpha}=\left\{\left(f_{1}^{\alpha}(t)+g_{1}^{\alpha}(t), f_{2}^{\alpha}(t)+g_{2}^{\alpha}\right.\right.$ $\left.(t)) \in \mathbb{R}^{2} \mid 0 \leq t \leq 2 \pi\right\}$.

(2) $A(-)_{p} B: \quad\left(A(-)_{p} B\right)^{\alpha}=\left\{\left(x_{\alpha}(t), y_{\alpha}(t)\right) \in \mathbb{R}^{2} \mid 0\right.$ $\leq t \leq 2 \pi\}$, where

$$
\begin{aligned}
& x_{\alpha}(t)= \begin{cases}f_{1}^{\alpha}(t)-g_{1}^{\alpha}(t+\pi), & \text { if } 0 \leq t \leq \pi, \\
f_{1}^{\alpha}(t)-g_{1}^{\alpha}(t-\pi), & \text { if } \pi \leq t \leq 2 \pi,\end{cases} \\
& y_{\alpha}(t)= \begin{cases}f_{2}^{\alpha}(t)-g_{2}^{\alpha}(t+\pi), & \text { if } 0 \leq t \leq \pi, \\
f_{2}^{\alpha}(t)-g_{2}^{\alpha}(t-\pi), & \text { if } \pi \leq t \leq 2 \pi .\end{cases}
\end{aligned}
$$

(3) $A(\cdot)_{p} B:\left(A(\cdot)_{p} B\right)^{\alpha}=\left\{\left(f_{1}^{\alpha}(t) \cdot g_{1}^{\alpha}(t), f_{2}^{\alpha}(t) \cdot g_{2}^{\alpha}(t)\right)\right.$ $\left.\in \mathbb{R}^{2} \mid 0 \leq t \leq 2 \pi\right\}$

(4) $A(/)_{p} B: \quad\left(A(/)_{p} B\right)^{\alpha}=\left\{\left(x_{\alpha}(t), y_{\alpha}(t)\right) \in \mathbb{R}^{2} \mid 0 \leq t\right.$ $\leq 2 \pi\}$, where

$$
\begin{array}{ll}
x_{\alpha}(t)=\frac{f_{1}^{\alpha}(t)}{g_{1}^{\alpha}(t+\pi)}, & (0 \leq t \leq \pi), \\
x_{\alpha}(t)=\frac{f_{1}^{\alpha}(t)}{g_{1}^{\alpha}(t-\pi)}, & (\pi \leq t \leq 2 \pi), \\
y_{\alpha}(t)=\frac{f_{2}^{\alpha}(t)}{g_{2}^{\alpha}(t+\pi)}, & (0 \leq t \leq \pi), \\
y_{\alpha}(t)=\frac{f_{2}^{\alpha}(t)}{g_{2}^{\alpha}(t-\pi)}, & (\pi \leq t \leq 2 \pi) .
\end{array}
$$

For $\alpha=0$ and $\alpha=1,\left(A(*)_{p} B\right)^{0}=\lim _{\alpha \longrightarrow 0^{+}}\left(A(*)_{p} B\right)^{\alpha}$ and $\left(A(*)_{p} B\right)^{1}=\lim _{\alpha \longrightarrow 1^{-}}\left(A(*)_{p} B\right)^{\alpha}$, where $*=+,-, \cdot, /$.

Theorem 2 (see [7]). Let $A=\left(a_{1}, x_{1}, b_{1}, y_{1}\right)^{2}$ and $B=\left(a_{2}, x_{2}, b_{2}, y_{2}\right)^{2}$ be two 2-dimensional triangular fuzzy numbers. Then, we have the following:

(1) $A(+)_{p} B=\left(a_{1}+a_{2}, x_{1}+x_{2}, b_{1}+b_{2}, y_{1}+y_{2}\right)^{2}$.

(2) $A(-)_{p} B=\left(a_{1}+a_{2}, x_{1}-x_{2}, b_{1}+b_{2}, y_{1}-y_{2}\right)^{2}$.

(3) $\left(A(\cdot)_{p} B\right)^{\alpha}=\left\{\left(x_{\alpha}(t), y_{\alpha}(t)\right) \mid 0 \leq t \leq 2 \pi\right\}$, where

$x_{\alpha}(t)=x_{1} x_{2}+\left(x_{1} a_{2}+x_{2} a_{1}\right)(1-\alpha) \cos t+a_{1} a_{2}(1-\alpha)^{2} \cos ^{2} t$,

$y_{\alpha}(t)=y_{1} y_{2}+\left(y_{1} b_{2}+y_{2} b_{1}\right)(1-\alpha) \sin t+b_{1} b_{2}(1-\alpha)^{2} \sin ^{2} t$.

(4) $\left(A(/)_{p} B\right)^{\alpha}=\left\{\left(x_{\alpha}(t), y_{\alpha}(t)\right) \mid 0 \leq t \leq 2 \pi\right\}$, where 


$$
\begin{aligned}
& x_{\alpha}(t)=\frac{x_{1}+a_{1}(1-\alpha) \cos t}{x_{2}-a_{2}(1-\alpha) \cos t} \\
& y_{\alpha}(t)=\frac{y_{1}+b_{1}(1-\alpha) \sin t}{y_{2}-b_{2}(1-\alpha) \sin t} .
\end{aligned}
$$

Therefore, $A(+)_{p} B$ and $A(-)_{p} B$ become 2-dimensional triangular fuzzy numbers, but $A(\cdot)_{p} B$ and $A(/)_{p} B$ are not 2dimensional triangular fuzzy numbers.

Theorem 3 (see [7]). Parametric operations on $\mathbb{R}^{2}$ in Definition 4 are the generalization of Zadeh's extension principles on $\mathbb{R}$.

$$
\mu_{A}(x, y, z)= \begin{cases}1-\sqrt{\frac{\left(x-x_{1}\right)^{2}}{a^{2}}+\frac{\left(y-y_{1}\right)^{2}}{b^{2}}+\frac{\left(z-z_{1}\right)^{2}}{c^{2}},} & \text { if } b^{2} c^{2}\left(x-x_{1}\right)^{2}+c^{2} a^{2}\left(y-y_{1}\right)^{2}+a^{2} b^{2}\left(z-z_{1}\right)^{2} \leq a^{2} b^{2} c^{2} \\ 0, & \text { otherwise, }\end{cases}
$$

where $a, b, c>0$ is called the 3-dimensional triangular fuzzy number and denoted by $\left(a, x_{1}, b, y_{1}, c, z_{1}\right)^{3}$.

$$
A_{\alpha}=\left\{(x, y, z) \in \mathbb{R}^{3} \mid\left(\frac{x-x_{1}}{a(1-\alpha)}\right)^{2}+\left(\frac{y-y_{1}}{b(1-\alpha)}\right)^{2}+\left(\frac{z-z_{1}}{c(1-\alpha)}\right)^{2} \leq 1\right\} .
$$

Definition 6. A 3-dimensional fuzzy number $A$ defined on $\mathbb{R}^{3}$ is called a convex fuzzy number if, for all $\alpha \in(0,1)$, the $\alpha$-cuts

$$
A_{\alpha}=\left\{(x, y, z) \in \mathbb{R}^{3} \mid \mu_{A}(x, y, z) \geq \alpha\right\},
$$

are convex subsets in $\mathbb{R}^{3}$.

Theorem 4 (see [8]). Let $A$ be a continuous convex fuzzy number defined on $\mathbb{R}^{3}$, and $A^{\alpha}=\{(x, y, z) \in$ $\left.\mathbb{R}^{3} \mid \mu_{A}(x, y, z)=\alpha\right\}$ be the $\alpha$-set of $A$. Then, for all $\alpha \in(0,1)$, there exist continuous functions $f_{1}^{\alpha}(s), f_{2}^{\alpha}(s, t)$, and $f_{3}^{\alpha}(s, t)(0 \leq s \leq 2 \pi,-(\pi / 2) \leq t \leq(\pi / 2))$, such that

$$
A^{\alpha}=\left\{\left(f_{1}^{\alpha}(s), f_{2}^{\alpha}(s, t), f_{3}^{\alpha}(s, t)\right) \in \mathbb{R}^{3} \mid 0 \leq s \leq 2 \pi,-\frac{\pi}{2} \leq t \leq \frac{\pi}{2}\right\} .
$$

Definition 7. Let $A$ and $B$ be two continuous convex fuzzy numbers defined on $\mathbb{R}^{3}$ and

$$
\begin{aligned}
& A^{\alpha}=\left\{\left(f_{1}^{\alpha}(s), f_{2}^{\alpha}(s, t), f_{3}^{\alpha}(s, t)\right) \in \mathbb{R}^{3} \mid 0 \leq s \leq 2 \pi,-\frac{\pi}{2} \leq t \leq \frac{\pi}{2}\right\}, \\
& B^{\alpha}=\left\{\left(g_{1}^{\alpha}(s), g_{2}^{\alpha}(s, t), g_{3}^{\alpha}(s, t)\right) \in \mathbb{R}^{3} \mid 0 \leq s \leq 2 \pi,-\frac{\pi}{2} \leq t \leq \frac{\pi}{2}\right\},
\end{aligned}
$$

be the $\alpha$-sets of $A$ and $B$, respectively. For $\alpha \in(0,1)$, we define that the parametric addition, parametric subtraction, parametric multiplication, and parametric division of two fuzzy numbers $A$ and $B$ are fuzzy numbers that have their $\alpha$-sets as follows:

(1) Parametric addition, $A(+)_{p} B$ :

$$
\left(A(+)_{p} B\right)^{\alpha}=\left\{\left(f_{1}^{\alpha}(s)+g_{1}^{\alpha}(s), f_{2}^{\alpha}(s, t)+g_{2}^{\alpha}(s, t), f_{3}^{\alpha}(s, t)+g_{3}^{\alpha}(s, t)\right) \in \mathbb{R}^{3} \mid 0 \leq s \leq 2 \pi,-\frac{\pi}{2} \leq t \leq \frac{\pi}{2}\right\}
$$

(2) Parametric subtraction, $A(-)_{p} B$ : 


$$
\begin{aligned}
& \left(A(-)_{p} B\right)^{\alpha}=\left\{\left(f_{1}^{\alpha}(s)-g_{1}^{\alpha}(s+\pi), f_{2}^{\alpha}(s, t)-g_{2}^{\alpha}(s+\pi, t), f_{3}^{\alpha}(s, t)-g_{3}^{\alpha}(s+\pi, t)\right) \in \mathbb{R}^{3} \mid 0 \leq s \leq \pi,-\frac{\pi}{2} \leq t \leq \frac{\pi}{2}\right\}, \\
& \left(A(-)_{p} B\right)^{\alpha}=\left\{\left(f_{1}^{\alpha}(s)-g_{1}^{\alpha}(s-\pi), f_{2}^{\alpha}(s, t)-g_{2}^{\alpha}(s-\pi, t), f_{3}^{\alpha}(s, t)-g_{3}^{\alpha}(s-\pi, t)\right) \in \mathbb{R}^{3} \mid \pi \leq s \leq 2 \pi,-\frac{\pi}{2} \leq t \leq \frac{\pi}{2}\right\} .
\end{aligned}
$$

(3) Parametric multiplication, $A(\cdot)_{p} B$ :

$$
\left(A(\cdot)_{p} B\right)^{\alpha}=\left\{\left(f_{1}^{\alpha}(s) \cdot g_{1}^{\alpha}(s), f_{2}^{\alpha}(s, t) \cdot g_{2}^{\alpha}(s, t), f_{3}^{\alpha}(s, t) \cdot g_{3}^{\alpha}(s, t)\right) \in \mathbb{R}^{3} \mid 0 \leq s \leq 2 \pi,-\frac{\pi}{2} \leq t \leq \frac{\pi}{2}\right\} .
$$

(4) Parametric division, $A(/)_{p} B$ :

$$
\begin{aligned}
& \left(A(/)_{p} B\right)^{\alpha}=\left\{\left(\frac{f_{1}^{\alpha}(s)}{g_{1}^{\alpha}(s+\pi)}, \frac{f_{2}^{\alpha}(s, t)}{g_{2}^{\alpha}(s+\pi, t)}, \frac{f_{3}^{\alpha}(s, t)}{g_{3}^{\alpha}(s+\pi, t)}\right) \in \mathbb{R}^{3} \mid 0 \leq s \leq \pi,-\frac{\pi}{2} \leq t \leq \frac{\pi}{2}\right\}, \\
& \left(A(/)_{p} B\right)^{\alpha}=\left\{\left(\frac{f_{1}^{\alpha}(s)}{g_{1}^{\alpha}(s-\pi)}, \frac{f_{2}^{\alpha}(s, t)}{g_{2}^{\alpha}(s-\pi, t)}, \frac{f_{3}^{\alpha}(s, t)}{g_{3}^{\alpha}(s-\pi, t)}\right) \in \mathbb{R}^{3} \mid \pi \leq s \leq 2 \pi,-\frac{\pi}{2} \leq t \leq \frac{\pi}{2}\right\} .
\end{aligned}
$$

For $\alpha=0$ and $\alpha=1,\left(A(*)_{p} B\right)^{0}=\lim _{\alpha \longrightarrow 0^{+}}\left(A(*)_{p} B\right)^{\alpha}$, and $\left(A(*)_{p} B\right)^{1}=\lim _{\alpha \longrightarrow 1^{-}}\left(A(*)_{p} B\right)^{\alpha}$, where $*=+,-, \cdot, /$.

Theorem 5 (see [8]). Let $A=\left(a_{1}, x_{1}, b_{1}, y_{1}, c_{1}, z_{1}\right)^{3}$ and $B=$ $\left(a_{2}, x_{2}, b_{2}, y_{2}, c_{2}, z_{2}\right)^{3}$ be two 3-dimensional triangular fuzzy numbers. Then, we have the following:
(1) $A(+)_{p} B=\left(a_{1}+a_{2}, x_{1}+x_{2}, b_{1}+b_{2}, y_{1}+y_{2}, c_{1}+c_{2}\right.$, $\left.z_{1}+z_{2}\right)^{3}$.

(2) $A(-)_{p} B=\left(a_{1}+a_{2}, x_{1}-x_{2}, b_{1}+b_{2}, y_{1}-y_{2}, c_{1}+c_{2}\right.$, $\left.z_{1}-z_{2}\right)^{3}$.

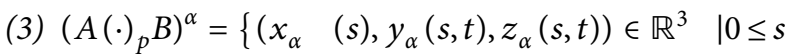
$\leq 2 \pi,-(\pi / 2) \leq t \leq(\pi / 2)\}$, where

$$
\begin{aligned}
x_{\alpha}(s) & =x_{1} x_{2}+\left(x_{1} a_{2}+x_{2} a_{1}\right)(1-\alpha) \cos s+a_{1} a_{2}(1-\alpha)^{2} \cos ^{2} s, \\
y_{\alpha}(s, t) & =y_{1} y_{2}+\left(y_{1} b_{2}+y_{2} b_{1}\right)(1-\alpha) \sin s \cos t+b_{1} b_{2}(1-\alpha)^{2} \sin ^{2} s \cos ^{2} t, \\
z_{\alpha}(s, t) & =z_{1} z_{2}+\left(z_{1} c_{2}+z_{2} c_{1}\right)(1-\alpha) \sin s \sin t+c_{1} c_{2}(1-\alpha)^{2} \sin ^{2} \sin ^{2} t .
\end{aligned}
$$

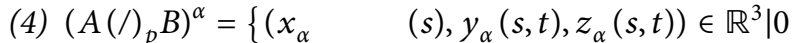
$\leq s \leq 2 \pi,-(\pi / 2) \leq t \leq(\pi / 2)\}$, where

$$
\begin{gathered}
x_{\alpha}(s)=\frac{x_{1}+a_{1}(1-\alpha) \cos s}{x_{2}-a_{2}(1-\alpha) \cos s} \\
y_{\alpha}(s, t)=\frac{y_{1}+b_{1}(1-\alpha) \sin s \cos t}{y_{2}-b_{2}(1-\alpha) \sin s \cos t} \\
z_{\alpha}(s, t)=\frac{z_{1}+c_{1}(1-\alpha) \sin s \sin t}{z_{2}-c_{2}(1-\alpha) \sin s \sin t} .
\end{gathered}
$$

Therefore, $A(+)_{p} B$ and $A(-)_{p} B$ become 3-dimensional triangular fuzzy numbers, but $A(\cdot)_{p} B$ and $A(/)_{p} B$ are not $3-$ dimensional triangular fuzzy numbers.
Theorem 6. Parametric operations on $\mathbb{R}^{3}$ in Definition 7 are the generalization of parametric operations on $\mathbb{R}^{2}$ in Definition 4, which are the generalization of Zadeh's max-min composition operations on $\mathbb{R}$.

Proof. Consider two 3-dimensional triangular fuzzy numbers $A=\left(a_{1}, x_{1}, b_{1}, y_{1}, c_{1}, 0\right)^{3} \quad$ and $B=\left(a_{2}, x_{2}, b_{2}, y_{2}, c_{2}, 0\right)^{3}$. By Theorem 5 ,

(1) $A(+)_{p} B=\left(a_{1}+a_{2}, x_{1}+x_{2}, b_{1}+b_{2}, y_{1}+y_{2}, c_{1}+c_{2}\right.$, $0)^{3}$.

(2) $A(-)_{p} B=\left(a_{1}+a_{2}, x_{1}-x_{2}, b_{1}+b_{2}, y_{1}-y_{2}, c_{1}+c_{2}\right.$, $0)^{3}$.

(3) $\left(A(\cdot)_{p} B\right)^{\alpha}=\left\{\left(x_{\alpha}(s), y_{\alpha}(s, t), z_{\alpha}(s, t)\right) \in \mathbb{R}^{3} \mid 0 \leq s \leq\right.$ $2 \pi,-(\pi / 2) \leq t \leq(\pi / 2)\}$, where 


$$
\begin{aligned}
x_{\alpha}(s) & =x_{1} x_{2}+\left(x_{1} a_{2}+x_{2} a_{1}\right)(1-\alpha) \cos s+a_{1} a_{2}(1-\alpha)^{2} \cos ^{2} s, \\
y_{\alpha}(s, t) & =y_{1} y_{2}+\left(y_{1} b_{2}+y_{2} b_{1}\right)(1-\alpha) \sin s \cos t+b_{1} b_{2}(1-\alpha)^{2} \sin ^{2} \operatorname{sos}^{2} t \\
z_{\alpha}(s, t) & =c_{1} c_{2}(1-\alpha)^{2} \sin ^{2} \sin ^{2} t
\end{aligned}
$$

(4) $\left(A(/)_{p} B\right)^{\alpha}=\left\{\left(x_{\alpha}(s), y_{\alpha}(s, t), z_{\alpha}(s, t)\right) \in \mathbb{R}^{3} \mid 0 \leq s \leq\right.$ $2 \pi,-(\pi / 2) \leq t \leq(\pi / 2)\}$, where

$$
\begin{gathered}
x_{\alpha}(s)=\frac{x_{1}+a_{1}(1-\alpha) \cos s}{x_{2}-a_{2}(1-\alpha) \cos s}, \\
y_{\alpha}(s, t)=\frac{y_{1}+b_{1}(1-\alpha) \sin s \cos t}{y_{2}-b_{2}(1-\alpha) \sin s \cos t}, \\
z_{\alpha}(s, t)=\frac{c_{1}(1-\alpha) \sin s \sin t}{-c_{2}(1-\alpha) \sin s \sin t}=-\frac{c_{1}}{c_{2}} .
\end{gathered}
$$

The intersections of these 3-dimensional triangular fuzzy numbers and $z=0$ are as follows:

(1) $A(+)_{p} B$ : note that

$$
\mu_{A(+)_{p} B}(x, y, z)=1-\sqrt{\left(\frac{x-x_{1}-x_{2}}{a_{1}+a_{2}}\right)^{2}+\left(\frac{y-y_{1}-y_{2}}{b_{1}+b_{2}}\right)^{2}+\left(\frac{z}{c_{1}+c_{2}}\right)^{2}} .
$$

If $z=0$,

$\mu_{A(+)_{P} B}(x, y, 0)=1-\sqrt{\left(\frac{x-x_{1}-x_{2}}{a_{1}+a_{2}}\right)^{2}+\left(\frac{y-y_{1}-y_{2}}{b_{1}+b_{2}}\right)^{2}}$.
Thus, the intersection is the 2-dimensional triangular fuzzy number $C=\left(a_{1}+a_{2}, x_{1}+x_{2}, b_{1}+b_{2}\right.$, $\left.y_{1}+y_{2}\right)^{2}$.

(2) $A(-)_{p} B$ : note that

$$
\mu_{A(-)_{p} B}(x, y, z)=1-\sqrt{\left(\frac{x-x_{1}+x_{2}}{a_{1}+a_{2}}\right)^{2}+\left(\frac{y-y_{1}+y_{2}}{b_{1}+b_{2}}\right)^{2}+\left(\frac{z}{c_{1}+c_{2}}\right)^{2}} .
$$

If $z=0$,

$\mu_{A(-)_{p} B}(x, y, 0)=1-\sqrt{\left(\frac{x-x_{1}+x_{2}}{a_{1}+a_{2}}\right)^{2}+\left(\frac{y-y_{1}+y_{2}}{b_{1}+b_{2}}\right)^{2}}$.
Thus, the intersection is the 2-dimensional triangular fuzzy number $D=\left(a_{1}+a_{2}, x_{1}-x_{2}, b_{1}+b_{2}\right.$, $\left.y_{1}-y_{2}\right)^{2}$.

(3) $A(\cdot)_{p} B$ : if $z=0, c_{1} c_{2}(1-\alpha)^{2} \sin ^{2} s \sin ^{2} t=0$. Thus, the intersection is a fuzzy number $E$ on $\mathbb{R}^{2}$ with $\alpha$-cut, not $\alpha$-set, $\left(A(\cdot)_{p} B\right)_{\alpha}=\left\{\left(x_{\alpha}(s), y_{\alpha}(s, 0)\right)\right.$ $\left.\in \mathbb{R}^{2} \mid 0 \leq s \leq 2 \pi\right\}$, where

$$
\begin{aligned}
x_{\alpha}(s) & =x_{1} x_{2}+\left(x_{1} a_{2}+x_{2} a_{1}\right)(1-\alpha) \cos s+a_{1} a_{2}(1-\alpha)^{2} \cos ^{2} s, \\
y_{\alpha}(s, 0) & =y_{1} y_{2}+\left(y_{1} b_{2}+y_{2} b_{1}\right)(1-\alpha) \sin s \cos 0+b_{1} b_{2}(1-\alpha)^{2} \sin ^{2} \operatorname{sos}^{2} 0 \\
& =y_{1} y_{2}+\left(y_{1} b_{2}+y_{2} b_{1}\right)(1-\alpha) \sin s+b_{1} b_{2}(1-\alpha)^{2} \sin ^{2} s .
\end{aligned}
$$




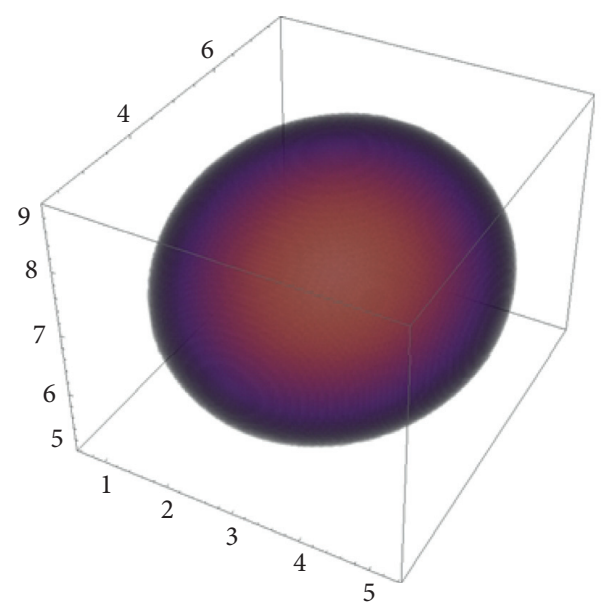

Figure 1: $A^{3}$.

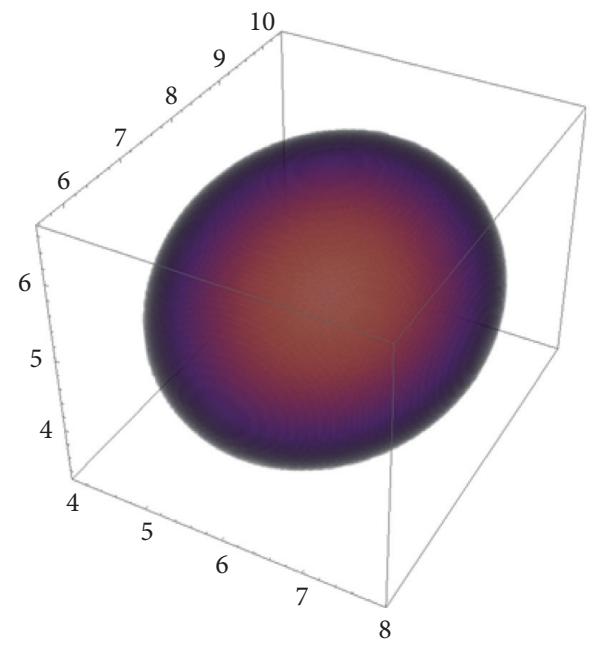

Figure 2: $B^{3}$.

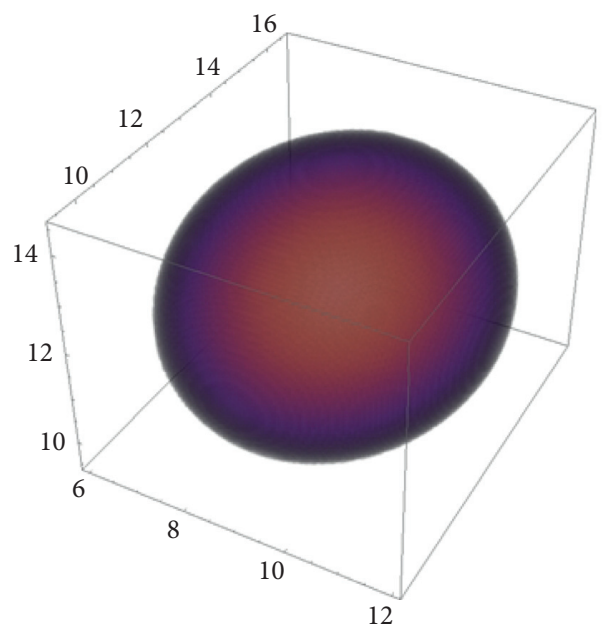

Figure 3: $(A+B)^{3}$.
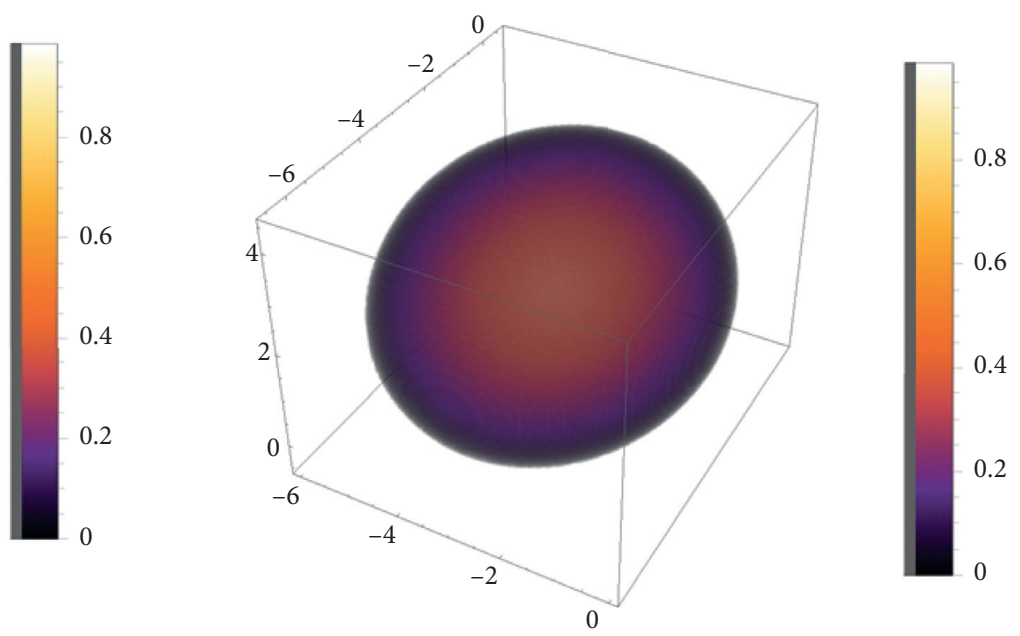

Figure 4: $(A-B)^{3}$.

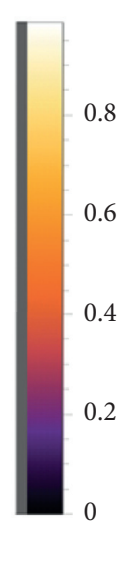

0.8

0.6

0.4

0.2

0

$$
\begin{array}{r}
x_{\alpha}(s)=\frac{x_{1}+a_{1}(1-\alpha) \cos s}{x_{2}-a_{2}(1-\alpha) \cos s} \\
y_{\alpha}(s, 0)=\frac{y_{1}+b_{1}(1-\alpha) \sin s}{y_{2}-b_{2}(1-\alpha) \sin s} .
\end{array}
$$




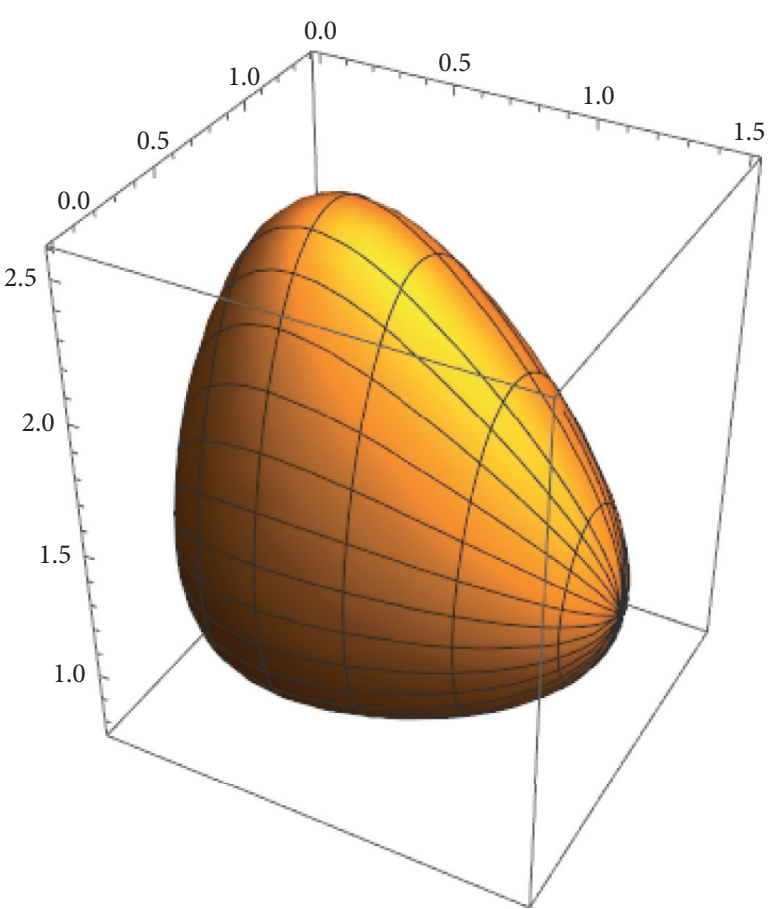

Figure 6: $(A / B)(1 / 2)$.

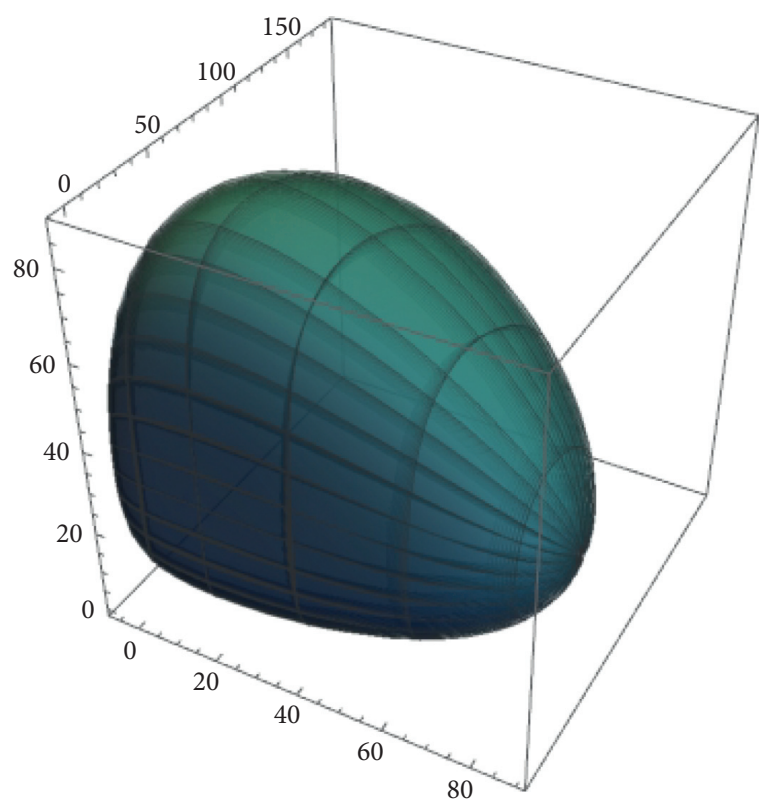

Figure 7: $(A \times B)^{3}$.

On the contrary, the intersection of 3-dimensional triangular fuzzy number $A=\left(a_{1}, x_{1}, b_{1}, y_{1}, c_{1}, 0\right)^{3}$ and $z=$ 0 is $G=\left(a_{1}, x_{1}, b_{1}, y_{1}\right)^{2}$, and the intersection of 3 -dimensional triangular fuzzy number $B=\left(a_{2}, x_{2}, b_{2}, y_{2}, c_{2}, 0\right)^{3}$ and $z=0$ is $H=\left(a_{2}, x_{2}, b_{2}, y_{2}\right)^{2}$. For two 2-dimensional triangular fuzzy numbers $G$ and $H$, the following results for parametric operations on $\mathbb{R}^{2}$ have been proven [7]:

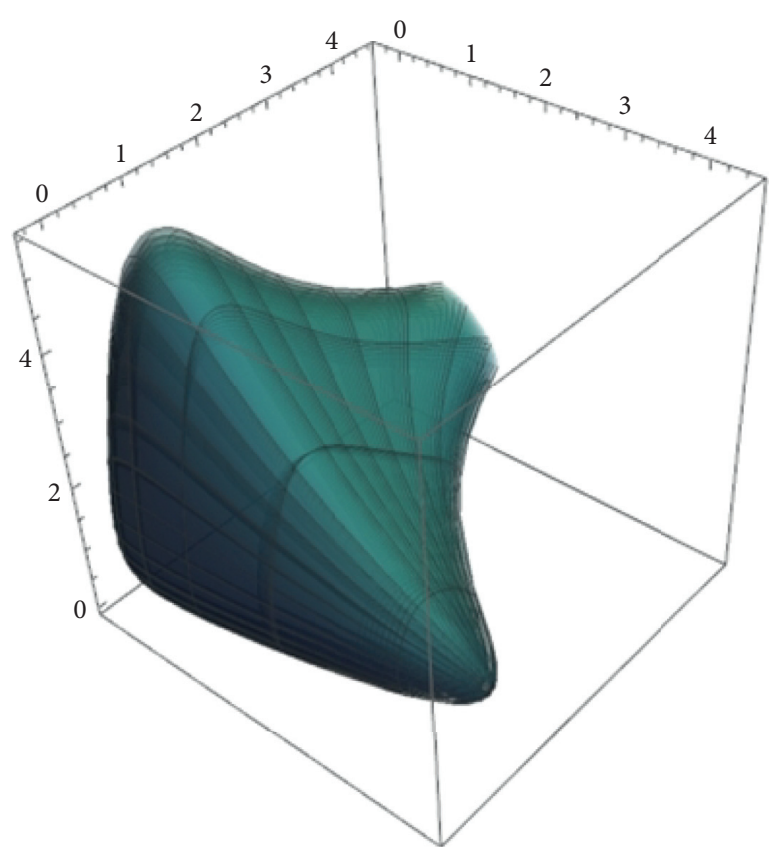

Figure 8: $(A / B)^{3}$.

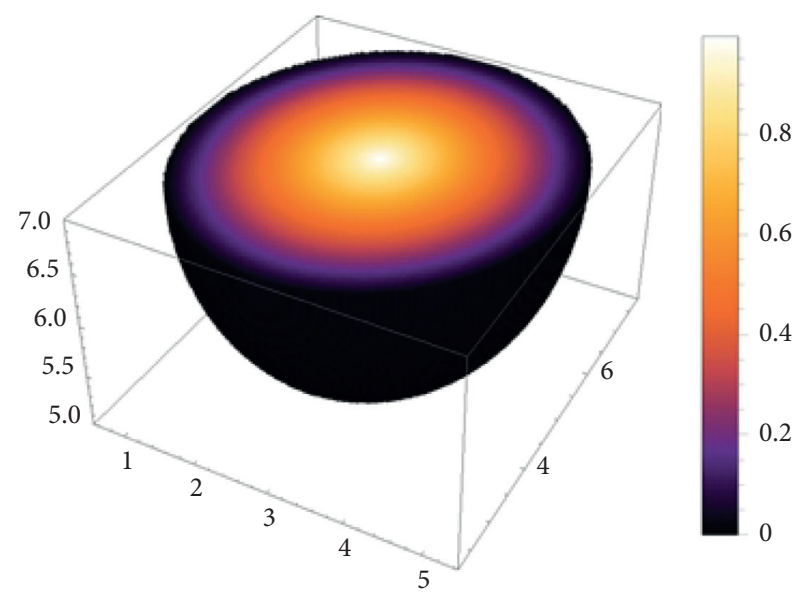

FIgURE 9: $A / 2$.

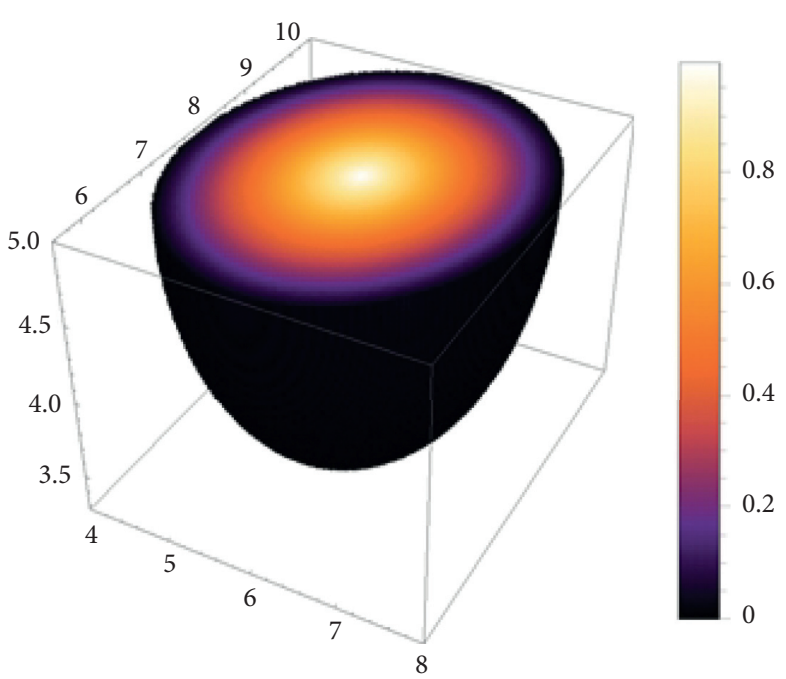

Figure 10: $B / 2$ 

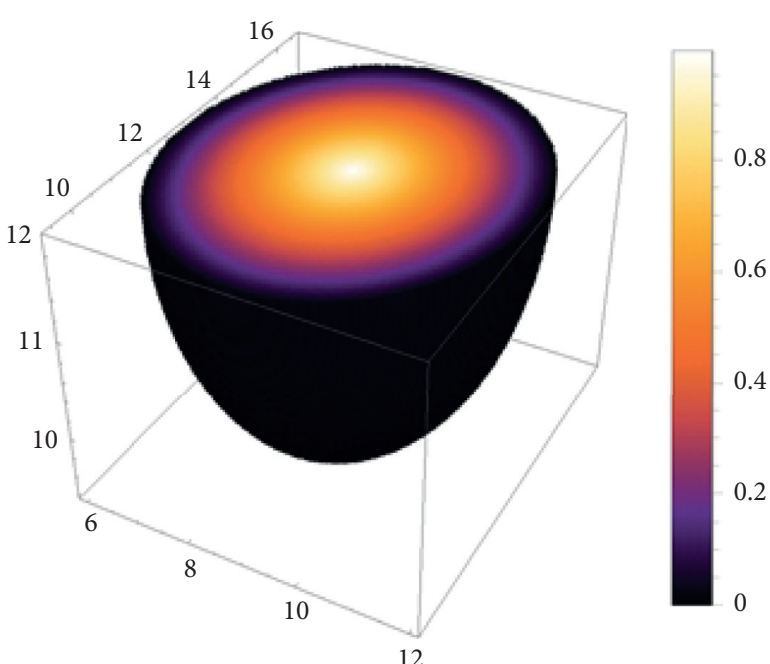

Figure 11: $(A+B) / 2$.
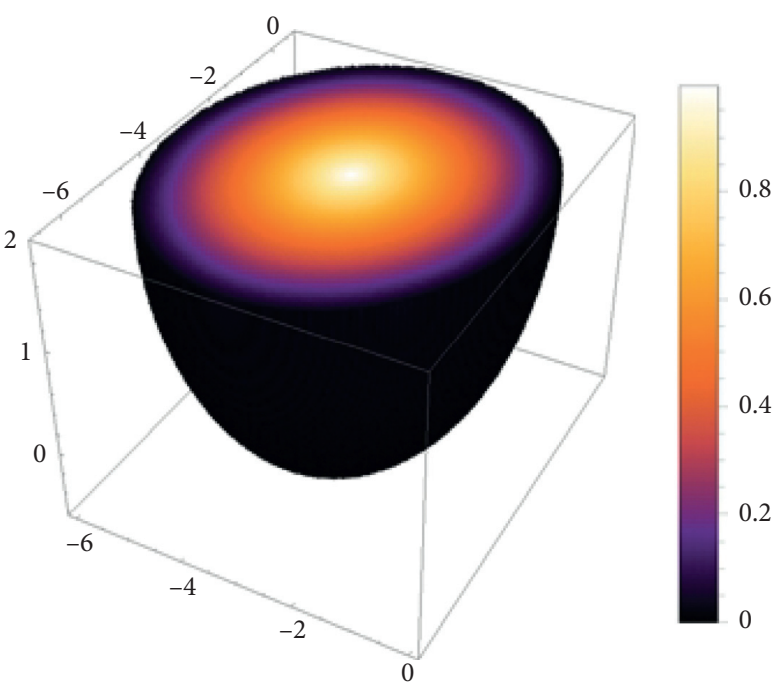

Figure 12: $(A-B) / 2$.

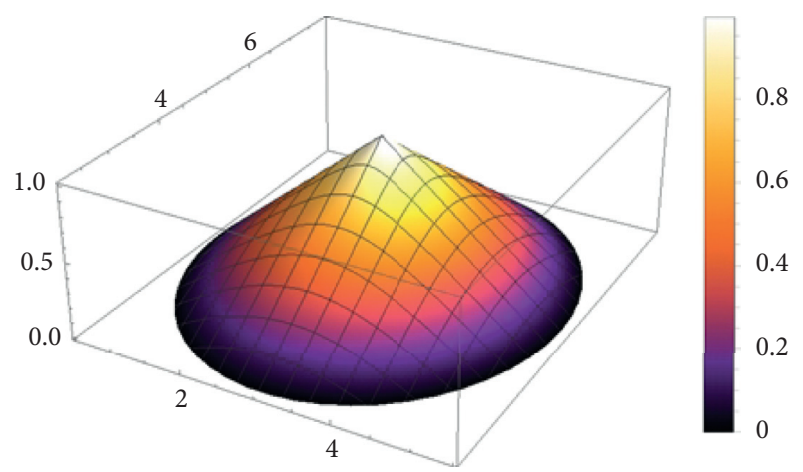

Figure 13: $A^{2}$.

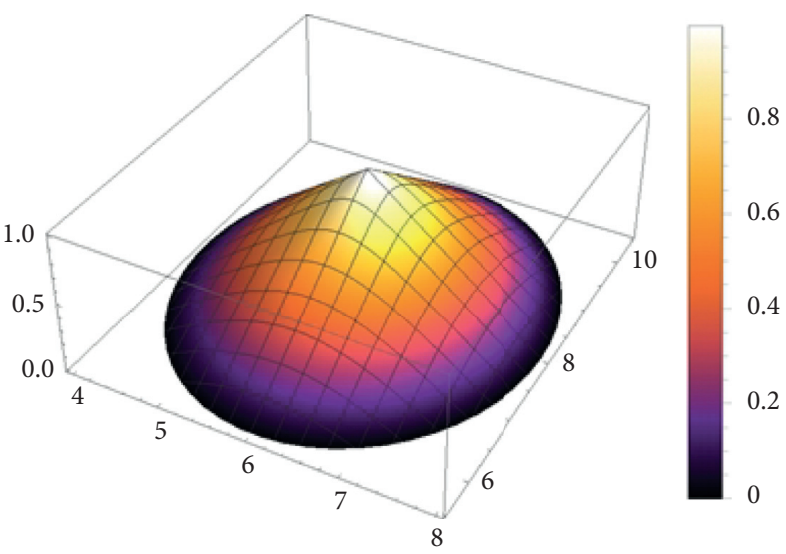

FIgURE 14: $B^{2}$.

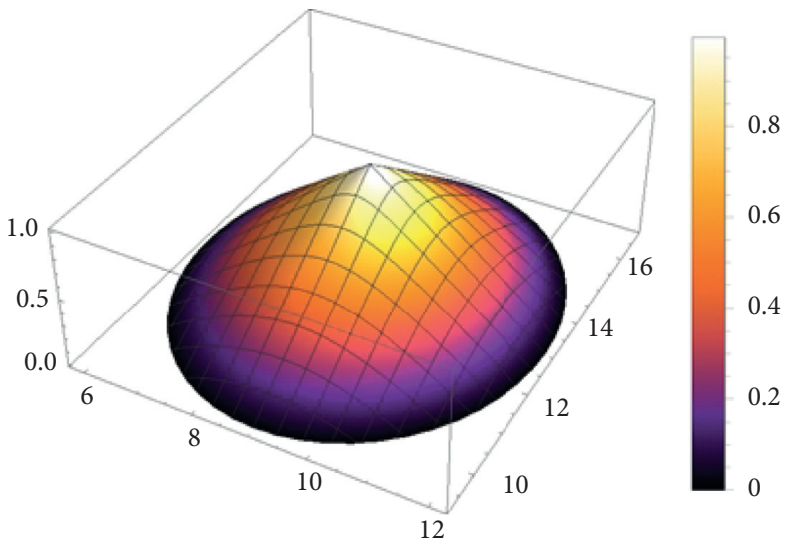

Figure 15: $(A+B)^{2}$.

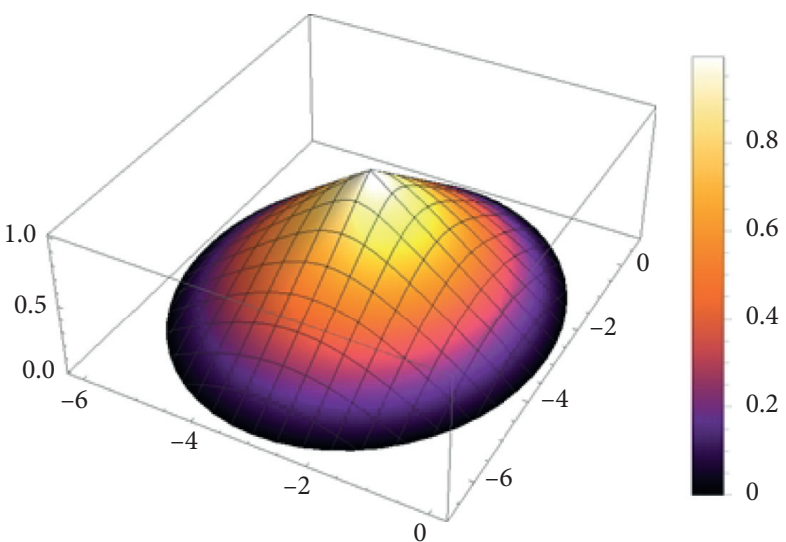

Figure 16: $(A-B)^{2}$.

$$
\begin{aligned}
G(+)_{p} H & =C, \\
G(-)_{p} H & =D, \\
G(\cdot)_{p} H & =E, \\
G(/)_{p} H & =F .
\end{aligned}
$$

The proof is complete. 


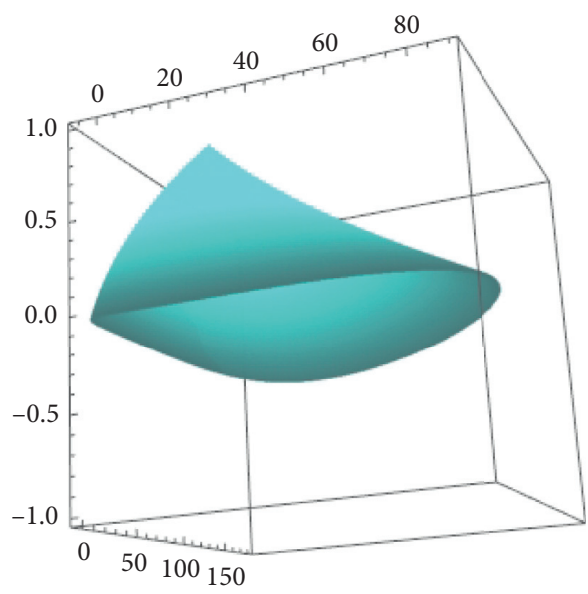

(a)

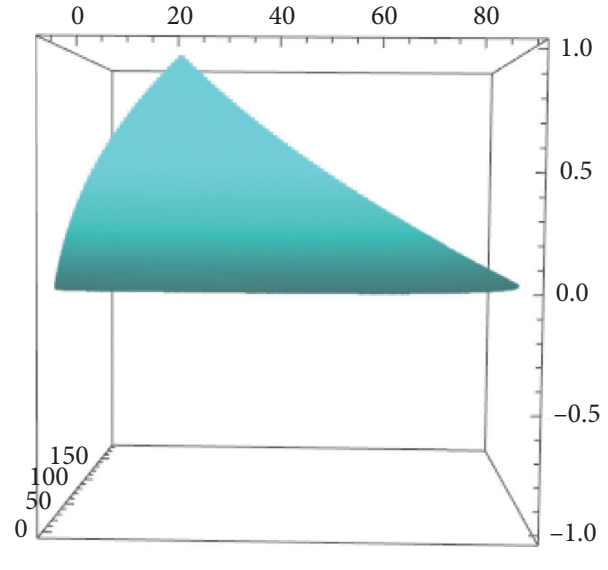

(b)

Figure 17: $(A \times B)^{2}$.

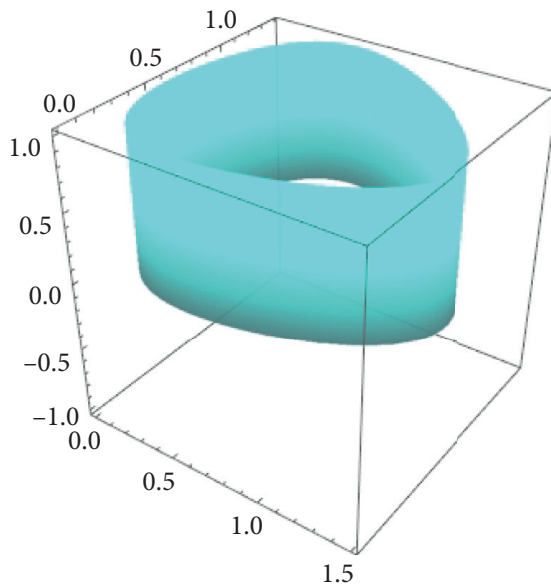

(a)

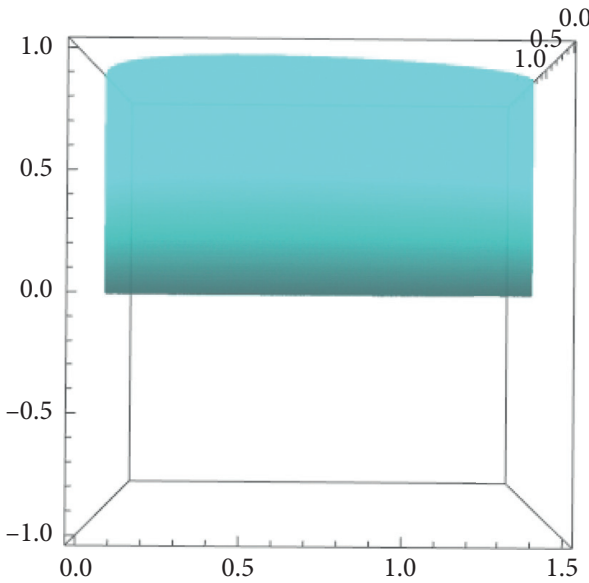

(b)

Figure 18: $(A / B)^{2}$.

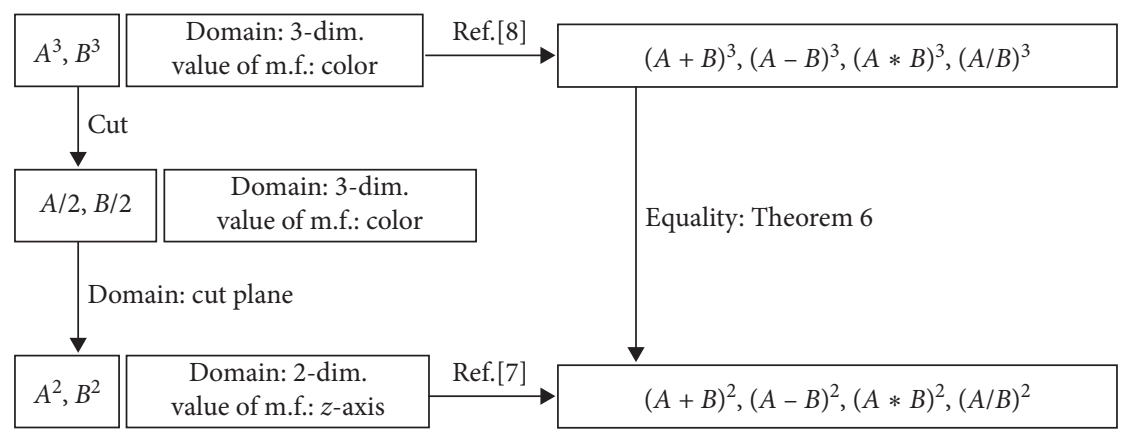

Figure 19: Summary.

\section{Conclusion}

In this section, by limiting the graph of the 3-dimensional result to the 2-dimensional case, we prove that the result expressed as a graph is consistent with the graph of the 2dimensional result.
For two 3-dimensional triangular fuzzy numbers $A=$ $(6,3,8,5,4,7)^{3}$ and $B=(4,6,6,8,3,5)^{3}$, the values of membership function are expressed using color density in Figures 1 and 2 , respectively. Zadeh's max-min operations expanded to 3 dimensions are expressed in Figures 3-6 as graphs. 
The graph of $A(\cdot) B$ and $A(/) B$ for $\alpha=1 / 2$ is Figures 5 and 6 , respectively. In Figures 7 and 8 , the graph for $A(\cdot) B$ and $A(/) B$ is the union of $\alpha(0 \leq \alpha \leq 1)$, respectively.

We cut the graph of $A^{3}, B^{3},(A+B)^{3}$, and $(A-B)^{3}$ by a perpendicular plane passing a vertex (Figures 9-12 ). The values of the membership function of each point on the cross section are expressed with color density.

We cut two 3-dimensional triangular fuzzy numbers $A^{3}$ and $B^{3}$ by a perpendicular plane passing a vertex and considered the cut plane as a domain. The value of the membership function for each point on the cut plane is expressed with color density. Figures 13 and 14 are the graphs expressing the value of the membership function defined in the plane as a 3-dimensional graph using the $z$-axis value, instead of expressing with color density. In this way, restricting the three-dimensional results (Figures 3-6) to two dimensions, we express the value of the membership function in a three-dimensional graph without using color density (Figures 15-18 ).

Consider the 2-dimensional triangular fuzzy numbers $A^{2}$ and $B^{2}$ represented by Figures 13 and 14, respectively. The result of Zadeh's max-min operators expanded to 2 dimensions can be found in [7]. When the results are graphed, it becomes clear that the graphs are identical to graphs in Figures 15-18. The commands in Mathematica for ten figures on page 7 and 8 are provided in appendix. Figure 19 summarizes what has been mentioned. In conclusion, therefore, the 3-dimensional result is naturally expanded to the 3 dimensions while satisfying the 2-dimensional result as it is, and it can be applied in this form. This paper will further study the extended applications of triangular fuzzy numbers [14-18].

\section{Appendix}

(i) $(: A 3:)$

DensityPlot3D $[1-\operatorname{Sqrt}[(x-3) 2 / 6+(y-5) 2 /$

$8+(z-7) 2 / 4],\{x, y, z\}$ in Ellipsoid $[\{3,5,7\}$, \{Sqrt [6], Sqrt[8], 2\}], PlotPoints->100, ColorFunction$>$ "SunsetColors", OpacityFunction->0.05, BoxRatios->\{Sqrt[6], Sqrt[8], 2\}, PlotLegends$>$ Automatic]

(ii) $(:(A+B) 3:)$

DensityPlot3D $[1-\operatorname{Sqrt}[(x-9) 2 / 10+(y-13) 2 /$

$14+(z-12) 2 / 7],\{x, y, z\}$ in Ellipsoid $[\{9,13,12\}$, $\{$ Sqrt[10], Sqrt[14], Sqrt[7]\}], PlotPoints->100, ColorFunction-> "SunsetColors", OpacityFunction- $>0.05$, BoxRatios- $>\{$ Sqrt[10], Sqrt[14], Sqrt[7]\}, PlotLegends- $>$ Automatic]

(iii) $(:(A * B) 1 / 2:)$

ParametricPlot3D $[\{18+24 \operatorname{Cos}[s]+6(\operatorname{Cos}[s]) 2$, $40+47 \quad \operatorname{Sin}[s] \operatorname{Cos}[t]+12 \quad(\operatorname{Sin}[s]) 2(\operatorname{Cos}[t]) 2$, $35+41 / 2 \operatorname{Sin}[s] \operatorname{Sin}[t]+3(\operatorname{Sin}[s]) 2(\operatorname{Sin}[t]) 2\},\{s, 0$, $2 \mathrm{Pi}\},\{t,-\mathrm{Pi} / 2, \mathrm{Pi} / 2\}$, Axes->True $]$

(iv) $(:(A / B) 3:)$ $\mathrm{g}\left[a_{-}\right] \backslash$ coloneq ParametricPlot $3 \mathrm{D}[\{(3+6(1-a) \operatorname{Cos}$ $[s]) /(6-4(1-a) \operatorname{Cos}[s]),(5+8(1-a) \operatorname{Sin}[s] \operatorname{Cos}[t]) /$ $(8-6(1-a) \quad \operatorname{Sin}[s] \operatorname{Cos}[t]),(7+4(1-a) \quad \operatorname{Sin}[s] \operatorname{Sin}$ $[t]) /(5-3(1-a) \operatorname{Sin}[s] \operatorname{Sin}[t])\},\{s, 0,2 \mathrm{Pi}\},\{t,-\mathrm{Pi} / 2$, $\mathrm{Pi} / 2\}$,PlotStyle- $>$ Directive[RGBColor $[0.2,0.5+a /$ $2,0.5+a / 2]$, Opacity[0.3]], BoxRatios- $>\{1,1,1\}]$; $\operatorname{tg}=$ Table $[\mathrm{g}[i],\{\mathrm{i}, 0,1.0,0.01\}]$;

Show $[\operatorname{tg}]$

(v) $(: A / 2:)$

reg1 $=$ ImplicitRegion $[0\{$ |textless $\}=(x-3) 2 /$

$6+(y-5) 2 / 8+(z-7) 2 / 4\{$ |textless $\}=1 \quad \& \& \quad z$ $\{$ textless $\}=7,\{x, y, z\}]$;

DensityPlot3D $[1-\operatorname{Sqrt}[(x-3) 2 / 6+((y-5) 2 /$ $8+((z-7) 2 / 4],\{x, y, z\}$ in reg1, PlotPoints->100, ColorFunction- $>$ "SunsetColors", Opacity Function- $>1$, BoxRatios- $>\{$ Sqrt[4], Sqrt[5], Sqrt[6]/2\}, PlotLegends- $>$ Automatic]

(vi) $(:(A+B) / 2:)$

reg1 $=$ ImplicitRegion $[0\{\backslash$ textless $\}=(x-9) 2 /$

$10+(y-13) 2 / 14+(z-12) 2 / 7\{\backslash$ textless $\}=1 \& \& z$

$\{\mid$ textless $\}=12,\{x, y, z\}]$;

DensityPlot3D $[1-\operatorname{Sqrt}[(x-9) 2 / 10+(y-13) 2 /$

$14+(z-12) 2 / 7],\{x, y, z\}$ in reg1, PlotPoints->100,

ColorFunction- $>$ "SunsetColors", Opacity-

Function- $>1$, BoxRatios- $>\{$ Sqrt[10], Sqrt[14], Sqrt [7]\}, PlotLegends->Automatic]

(vii) $(: A 2:)$

Plot3D $[1-\operatorname{Sqrt}[(x-3) 2 / 6+(y-5) 2 / 8],\{x, y\}$ in Ellipsoid $[\{3,5\},\{$ Sqrt[6], Sqrt[8]\}], PlotPoints- $>50$, ColorFunction- $>$ "SunsetColors", BoxRatios- $>$ \{Sqrt[6], Sqrt [8], 1\}, PlotLegends->Automatic]

(viii) $(:(A+B) 2:)$

Plot3D $[1-\operatorname{Sqrt}[(x-9) 2 / 10+(y-13) 2 / 14],\{x, y\}$ in Ellipsoid[\{9, 13\}, \{Sqrt[10], Sqrt[14]\}], PlotPoints- $>50$, ColorFunction- "SunsetColors", BoxRatios- $>\{$ Sqrt[6], Sqrt[8], 1\}, PlotLegends$>$ Automatic]

(ix) $(:(A * B) 2:)$

$\mathrm{g}\left[a_{-}\right] \backslash$ coloneq ParametricPlot3D $[\{18+48(1-a)$ $\operatorname{Cos}[s]+24(1-\mathrm{a}) 2(\operatorname{Cos}[s]) 2, \quad 40+94(1-a) \operatorname{Sin}$ $[s]+48(1-a) 2(\operatorname{Sin}[s]) 2, a\},\{s, 0,2 \mathrm{Pi}\}$, PlotStyle$>$ Directive[RGBColor $[0.3,0.5+a, 0.5+a]$, Opacity[0.3]], BoxRatios- $>\{1,1,1\}]$;

$\operatorname{tg}=$ Table $[g[i],\{i, 0,1.0,0.001\}]$;

Show $[\operatorname{tg}]$

(x) $(:(A / B) 2:)$

$\mathrm{g}\left[a_{-}\right] \backslash$ coloneq ParametricPlot3D $[\{(3+3 \operatorname{Cos}[\mathrm{s}]) /$ $(6-2 \operatorname{Cos}[s]),(5+4 \operatorname{Sin}[s]) /(8-3 / 2 \operatorname{Sin}[s]), a\},\{s, 0$, $2 \mathrm{Pi}$, PlotStyle- $>$ Directive[RGBColor $[0.3,0.5+a$, $0.5+a]$, Opacity[0.3]], BoxRatios- $>\{1,1,1\}]$; $\operatorname{tg}=$ Table $[\mathrm{g}[i],\{i, 0,1.0,0.001\}]$;

Show $[\operatorname{tg}]$ 


\section{Data Availability}

No data were used to support this study.

\section{Conflicts of Interest}

The author declares that there are no conflicts of interest regarding the publication of this article.

\section{Acknowledgments}

This research was supported by the 2021 scientific promotion program funded by Jeju National University.

\section{References}

[1] M. Bhowmik, M. Pal, and A. Pal, "Circulant triangular fuzzy number matrices," Journal of Physical Sciences, vol. 12, pp. 141-154, 2008.

[2] C. Kang and Y. S. Yun, "An extension of Zadeh's max-min composition operator," International Journal of Mathematical Analysis, vol. 9, no. 41, pp. 2029-2035, 2015.

[3] A. Priyanga and R. A. Myvizhi, "Two new operators on triangular fuzzy matrices," International Journal of Research in Engineering, Science and Management, vol. 2, no. 1, 2019.

[4] A. K. Shyamal and M. Pal, "Triangular fuzzy matrices," Iranian Journal of Fuzzy Systems, vol. 4, no. 1, pp. 75-87, 2007.

[5] X. Zhang, W. Ma, and L. Chen, "New similarity of triangular fuzzy number and its application," Recent Advances in Information Technology, vol. 2014, Article ID 215047, 7 pages, 2014.

[6] C. Kim and Y. S. Yun, "Zadeh's extension principle for 2dimensional triangular fuzzy numbers," Journal of Korean Institute of Intelligent Systems, vol. 25, no. 2, pp. 197-202, 2015.

[7] J. Byun and Y. S. Yun, "Parametric operations for two fuzzy numbers," Communications of the Korean Mathematical Society, vol. 28, no. 3, pp. 635-642, 2013.

[8] Y. S. Yun, “A Zadeh's max-min composition operator for 3dimensional triangular fuzzy number," Journal of Intelligent \& Fuzzy Systems, vol. 39, no. 3, pp. 3783-3793, 2020.

[9] A. Das, M. Pal, and M. Bhowmik, "Permanent of intervalvalued and triangular number fuzzy matrices," Annals of Fuzzy Mathematics and Informatics, vol. 10, no. 3, pp. 381395, 2015.

[10] H. J. Zimmermann, Fuzzy Set Theory- and its Applications, Kluwer-Nijhoff Publishing, Boston-Dordrecht-Lancaster, PA, USA, 1985.

[11] L. A. Zadeh, "The concept of a linguistic variable and its application to approximate reasoning-I," Information Sciences, vol. 8, no. 3, pp. 199-249, 1975.

[12] L. A. Zadeh, "-The concept of a linguistic variable and its application to approximate reasoning-II," Information Sciences, vol. 8, no. 4, pp. 301-357, 1975.

[13] L. A. Zadeh, "-The concept of a linguistic variable and its application to approximate reasoning-III," Information Sciences, vol. 9, no. 1, pp. 43-80, 1975.

[14] D. Ahmed and B. Dai, "Picture fuzzy rough set and rough picture fuzzy set on two different universes and their applications," Journal of Mathematics, vol. 2020, Article ID 8823580, 17 pages, 2020.

[15] H. Garg, R. Sujatha, D. Nagarajan, J. Kavikumar, and J. Gwak, "Evidence theory in picture fuzzy set environment," Journal of Mathematics, vol. 2021, Article ID 9996281, 8 pages, 2021.
[16] S. Shahzadi, M. Sarwar, and M. Akram, "Decision-making approach with fuzzy type-2 soft graphs," Journal of Mathematics, vol. 2020, Article ID 8872446, 25 pages, 2020.

[17] M. Gr. Voskoglou, "An application of triangular fuzzy numbers to learning assessment," Journal of Physical Sciences, vol. 20, pp. 63-79, 2015.

[18] M. G. Voskoglou and I. Ya. Subbotin, "Application of fuzzy numbers to assessment of human skills," International Journal of Fuzzy System Applications, vol. 6, no. 3, pp. 59-73, 2017. 